\title{
Frontières
}

\section{Le calme après la tempête}

Du choc à la résilience

\section{Josianne Crête}

Volume 22, numéro 1-2, automne-printemps 2009-2010

Résilience et deuil

URI : https://id.erudit.org/iderudit/045025ar

DOI : https://doi.org/10.7202/045025ar

Aller au sommaire du numéro

\section{Éditeur(s)}

Université du Québec à Montréal

ISSN

1180-3479 (imprimé)

1916-0976 (numérique)

Découvrir la revue

Citer cet article

Crête, J. (2009). Le calme après la tempête : du choc à la résilience. Frontières, 22(1-2), 35-41. https://doi.org/10.7202/045025ar

\section{Résumé de l'article}

Puisque l'annonce d'un diagnostic de déficience physique a des impacts tant sur la personne qui l'apprend que sur ses proches, plusieurs études se sont penchées sur ce que cette annonce fait vivre à ces derniers. Par contre, les étapes qu'ils traversent pour s'adapter à la nouvelle situation sont moins connues que celles vécues par la personne atteinte. Une recension des écrits a permis d'identifier deux processus traversés par les proches d'une personne atteinte à la suite de l'annonce du diagnostic. Le premier est chronologique et comporte quatre étapes : avant l'annonce, le choc suivant l'annonce, la réadaptation et après la réadaptation. Le deuxième est dynamique, systémique et évolutif, il s'agit du processus de deuil. Différentes issues à ces processus sont possibles ; elle peut être résiliente, haoméostatique, mésadaptée ou dysfonctionnelle. L'identification de ces processus permet d'identifier des pistes d'intervention.
Ce document est protégé par la loi sur le droit d'auteur. L'utilisation des services d'Érudit (y compris la reproduction) est assujettie à sa politique d'utilisation que vous pouvez consulter en ligne.

https://apropos.erudit.org/fr/usagers/politique-dutilisation/ 


\section{Résumé}

Puisque l'annonce d'un diagnostic de déficience physique a des impacts tant sur la personne qui l'apprend que sur ses proches, plusieurs études se sont penchées sur ce que cette annonce fait vivre à ces derniers. Par contre, les étapes qu'ils traversent pour s'adapter à la nouvelle situation sont moins connues que celles vécues par la personne atteinte. Une recension des écrits a permis d'identifier deux processus traversés par les proches d'une personne atteinte à la suite de I'annonce du diagnostic. Le premier est chronologique et comporte quatre étapes: avant l'annonce, le choc suivant l'annonce, la réadaptation et après la réadaptation. Le deuxième est dynamique, systémique et évolutif, il s'agit du processus de deuil. Différentes issues à ces processus sont possibles; elle peut être résiliente, homéostatique, mésadaptée ou dysfonctionnelle. L'identification de ces processus permet d'identifier des pistes d'intervention.

Mots clés: processus de deuil processus d'adaptation - déficience physique - résilience - proches.

\begin{abstract}
Knowing that breaking the news of a physical handicap diagnosis has impacts both on the person with the handicap and on his or her relatives, many studies researched what were the impacts of that news on the latter. Nevertheless, the stages these relatives go through to adapt to the new situation are not as well known as the stages the person experiences him- or herself. A review of the literature enabled us to identify two processes the relatives of a person with a physical handicap go through. The first one is chronological and has four stages: before the news, the shock after the news, the rehabilitation period and after the rehabilitation. The second is dynamic, systemic and changes with time; it is the grieving process. Many outcomes are possible after theses processes; it can be resilient, homeostatic, maladaptive or dysfunctional. The identification of these processes enables us to suggest some intervention techniques.
\end{abstract}

Keywords: grieving process adaptation process - physical handicap - resiliency - relatives.

\section{LE CALME APRÈS LA TEMPÊTE Du choc à la résilience}

\section{Josianne Crête, \\ travailleuse sociale CRLB, membre clinicienne du CRIR et du REPAR, étudiante à la maîtrise en service social, Université de Montréal.}

Dans le domaine de la réadaptation en déficience physique (DP), il existe un consensus sur le fait que l'annonce du diagnostic entraîne des impacts autant pour la personne atteinte que pour ses proches. En effet, de nombreuses études se sont penchées sur les impacts de l'annonce d'un diagnostic de DP sur les proches de la personne atteinte (Lefebvre et al., 2007; O'Connell et al., 2004; Mann, 2002; Degeneffe, 2001) et démontrent qu'il importe de les soutenir eux aussi afin de favoriser le mieux-être de chaque membre du système familial. Cependant, nous constatons que les étapes qu'ils traversent pour s'adapter à la nouvelle situation ont été moins documentées que celles parcourues par la personne atteinte.

Grâce à une recension des écrits ${ }^{1}$, il a été possible d'identifier deux processus que traversent les proches de la personne atteinte après avoir appris le diagnostic de DP. Le premier est un processus chrono- logique qui suit quatre étapes: la période avant l'annonce du diagnostic, le choc à la suite de cette annonce, la période de réadaptation et celle après la réadaptation. Le deuxième processus ayant été relevé dans les écrits est le processus de deuil qui est dynamique, systémique et évolutif et qui traverse toutes les étapes chronologiques suivant l'annonce de la DP.

Dans le présent texte, après avoir détaillé les étapes de ces deux processus, il sera question de leur issue possible. Cette issue peut être tant dysfonctionnelle, mésadaptée, homéostatique que résiliente. Ensuite, des pistes d'intervention tenant compte de ces informations seront proposées.

\section{LE PROCESSUS D'ADAPTATION DES PROCHES}

Bien que chaque personne et chaque système familial vivent différemment l'annonce d'un diagnostic de DP, tous ont à traverser certaines étapes dans un ordre précis. En effet, tout système familial dont un membre est atteint d'une DP est d'abord un système, ce qui est la nature même de 
la famille. Ensuite, lors de l'annonce du diagnostic, le système familial est perturbé dans son organisation. Par la suite, des services temporaires de réadaptation sont offerts afin de répondre à certains des besoins qu'expérimentent les proches. Pour terminer, ces services prennent fin et le système familial doit retrouver un équilibre, plus ou moins précaire, pour répondre à ses besoins. Ces quatre étapes représentent le processus d'adaptation des proches, que nous détaillerons maintenant.

\section{ÉTAPE 1: LE CALME AVANT LA TEMPÊTE}

Selon l'approche systémique, un système familial répond à certaines caractéristiques. En effet, selon Destaillats et ses collègues (2007), un système est «un ensemble d'éléments en interaction, auto-éco-organisé, autorégulé, tendant vers un but et qui échange, pour survivre et se transformer au fil du temps, de la matière, de l'énergie et des informations ». Le système familial a donc une fonction structurante interne qui se confond avec son existence même. Il assure un ordre relationnel qui crée une stabilité et une prévisibilité dont bénéficient ses membres.

Toujours selon Destaillats et ses collègues (2007), le système familial se décrit aussi comme une organisation relationnelle spécifique, originale et complexe. Il est formé de sous-systèmes de niveaux logiques différents basés sur des règles de fonctionnement qui les rattachent aux règles des groupes d'appartenance. En tant que groupe d'appartenance, le système familial permet à chacun de ses membres de fonder son identité sur le fait d'en être issus (pôle d'appartenance), sans gommer son originalité (pôle d'identité). Ces deux pôles, appartenance et identité, peuvent tant être opposés que complémentaires et la relation entre eux se nomme la boucle de la réflexivité.

\section{ÉTAPE 2: À LA SUITE DE L'ANNONCE DU DIAGNOSTIC}

Chaque famille est unique et a sa propre idée de ce qu'est et de ce que devrait être une famille avec ses rôles et ses règles de fonctionnement. L'annonce de la DP perturbe cette organisation et provoque une crise dans le système, qui ne peut plus se fier à ses repères habituels. Toutefois, cette perturbation est différente pour chaque membre de chaque système familial étant donné que chacun la perçoit et $\mathrm{y}$ réagit différemment. Aussi, l'effet de surprise de l'apparition de toute DP ajoute un stress important à l'événement luimême. Destaillats et ses collègues (2007) décrivent deux types de crise pouvant survenir au sein d'un système familial à la suite de l'annonce d'un diagnostic de
DP chez un de ses membres : la crise autoréférentielle et la crise hétéroréférentielle.

La crise autoréférentielle survient du fait que, habituellement, les ressources adaptatives sont pensées à l'intérieur du système familial et que, avec l'apparition de la DP, la compétence pour penser ces ressources est attribuée à des acteurs extérieurs, soit les professionnels de la santé. Cette extériorisation du contrôle entraîne une disqualification symbolique des rôles de chacun des membres du système familial et une perte de contrôle sur leur histoire familiale. Cela déstabilise le système et le fait douter de son modèle relationnel et de ses compétences. Aussi, cela remet en question les règles relationnelles et fait augmenter le niveau d'incertitude et de souffrance individuelle et collective.

En plus de vivre une crise interne (autoréférentielle) en se remettant lui-même en question, le système familial est confronté à des remises en question qui lui viennent de l'extérieur, ce qui entraîne la crise hétéroréférentielle. Cette crise est causée par le fait que le système familial et les institutions soignantes utilisent des approches qui s'opposent à plusieurs niveaux, opposition qui renforce les doutes du système familial face à ses capacités d'adaptation. Ainsi, d'un côté, il y a un groupe d'appartenance où le type de relation est choisi et où l'un des membres se trouve affecté d'une DP, alors que, de l'autre côté, il y a un groupe d'inclusion où les relations sont imposées en termes de patient et de soignant. De plus, la famille se compare aux membres de l'équipe soignante et remet son modèle en question alors qu'elle vit une période de crise interne (autoréférentielle), en même temps que l'équipe soignante tient un discours qui disqualifie, habituellement de façon implicite, le modèle relationnel de la famille. Cela se fait principalement de deux façons : 1) soit que l'équipe traitante dissimule ses doutes sur les limites de ses propres modèles théoriques et ses compétences, 2) soit qu'elle propose des modèles relationnels différents de ceux utilisés par le système familial, suggérant ainsi que le modèle actuel ou antérieur à l'annonce de la DP n'est pas adéquat. Cette dynamique renforce les doutes que ressent la famille et se nomme la crise hétéroréférentielle.

Ces deux types de crise se traduisent par des impacts concrets sur les proches: impacts individuels (anxiété, incompréhension, colère, culpabilité, fatigue, perte d'identité, etc.), familiaux (changement de rôles, problèmes financiers, apparition de conflits dans la famille, etc.) et environnementaux (isolement de la famille, bris de relation, marginalisation, etc.).

Pour faire face à ces impacts, les proches doivent utiliser différentes stra- tégies d'adaptation, qui peuvent être émotives (découragement, diminution de l'estime de soi, colère, déni, etc.), corporelles ou psychosomatiques (développement de maladies ou de problèmes cognitifs) ou comportementales (surprotéger, infantiliser, s'isoler, se désinvestir, se priver de plaisir, devenir agressif, etc.). Les membres de la famille peuvent aussi utiliser des stratégies positives, entre autres en ayant une attitude positive et des attentes réalistes par rapport à l'avenir de leur famille.

Il est à noter que les stratégies utilisées vont différer selon la nature du diagnostic. En effet, s'il s'agit d'un diagnostic annonçant une récupération graduelle, bien que partielle, des capacités fonctionnelles, comme pour les personnes ayant subi un traumatisme craniocérébral ou une lésion médullaire, les implications seront différentes pour les proches de celles découlant d'un diagnostic sous-entendant une fragilisation de la santé, comme c'est le cas chez les personnes ayant subi un accident vasculaire cérébral, ou sous-entendant une mort imminente, comme chez les personnes souffrant de sclérose latérale amyotrophique. Malgré cela, comme le relèvent plusieurs auteurs (Lefebvre et al. 2007 ; Lacerte et al., 2002; Rolland, 1994), la nature de l'événement déstabilisant le système familial, soit la nature du diagnostic de DP, à lui seul n'est pas suffisant pour prédire le processus que suivront les personnes le vivant. La perception $\mathrm{du}$ diagnostic qu'a chacun des membres du système familial et la signification que prend ce diagnostic pour le système jouent un rôle plus central dans la détermination du processus qu'il vivra après un tel choc.

\section{ÉTAPE 3: LA RÉADAPTATION}

Une fois le diagnostic annoncé, le système familial s'intègre habituellement dans un suivi en réadaptation. Les diagnostics varient, les traitements diffèrent, les cheminements aussi, mais certains besoins sont constants, perçus par une majorité de proches de personnes ayant une DP. Les études consultées classent ces besoins en trois catégories: les besoins d'informations, les besoins relationnels de soutien et les besoins en soins et en services.

Les études montrent que le manque d'information contribue à un sentiment d'insécurité, d'impuissance, à une perception irréaliste de la situation, à une augmentation des coûts pour le système de santé et à l'insatisfaction des personnes et des proches en regard de leur qualité de vie (Lefebvre et al., 2005; Fallowfield et al., 2002; Coulter, 1998; Wiles et al., 1998). Il est donc important de répondre à ce besoin en leur donnant de l'information sur tout ce qui entoure la DP. 
Les proches ont aussi des besoins relationnels de soutien, soit le besoin d'être partie prenante des soins de la personne. Afin d'obtenir le soutien psychologique dont ils ont besoin pour bien gérer leurs émotions, ils doivent souvent puiser dans leurs ressources personnelles et environnementales. Ces ressources peuvent être naturelles (famille, amis, collègues ou autres) ou professionnelles (intervenants). Bien sûr, il faut parallèlement maximiser le bien-être de la personne atteinte d'une DP, puisque le bien-être des proches est tributaire du bien-être de la personne atteinte.

Afin de diminuer leur fardeau objectif, les proches ont besoin de recevoir de l'aide et des services concrets, tant par leur réseau naturel que par le réseau professionnel. Ainsi, il faut les aider à identifier et à faire appel à des ressources pouvant combler leurs besoins d'aide à domicile (aide ménagère, popote roulante, etc.), leur offrir du répit, les accompagner dans différentes démarches administratives et combler leurs besoins financiers. Ce soutien social plus adapté à leurs besoins leur permettra d'avoir plus de temps pour eux.

L'expérience clinique et les études montrent que les proches ont un double rôle en réadaptation (Rochette et al., 2007). Ils ont d'abord un rôle de «client», étant donné qu'ils ont des besoins physiques et émotionnels causés par la DP, et aussi un rôle de "proche intervenant», parce qu'ils doivent prodiguer des soins à la personne et s'impliquer dans sa réadaptation. Afin de répondre adéquatement aux besoins des proches, il importe de garder en tête cette dualité entre le besoin de s'investir dans les soins de l'autre (rôle de proche intervenant) et celui de recevoir les soins dont ils ont eux-mêmes besoin (rôle de client).

\section{ÉTAPE 4 : APRÈS LA RÉADAPTATION}

Tout au long de la réadaptation, les intervenants proposent des outils au système familial afin que celui-ci retrouve un équilibre relationnel malgré la déstabilisation causée par l'annonce de la DP. Par contre, quand la réadaptation se termine, les services changent et les proches n'ont plus le même soutien professionnel qu'avant. Divers scénarios deviennent alors possibles. Parfois, les services cessent complètement et le réseau social dit «naturel » prend la relève, d'autres fois, ils sont maintenus par un suivi dans la communauté (CLSC, organismes communautaires, etc.) ou encore, ils sont remplacés par un suivi interne (hébergement). Le choix du scénario à privilégier est influencé par plusieurs facteurs, comme l'état de la personne atteinte d'une DP, la nature évolutive ou non de sa DP, l'état de ses proches et leur capacité et disponibilité à offrir le soutien nécessaire, en plus de facteurs sociétaux comme la disponibilité des services nécessaires.

Par ailleurs, les études démontrent que, malgré les différents scénarios possibles, les besoins à long terme des proches demeurent les mêmes. En effet, il revient souvent aux familles d'assumer en partie les soins à prodiguer, sans que celles-ci aient les ressources suffisantes et les connaissances nécessaires pour y parvenir (O'Connell et al., 2004 ; Degeneffe, 2001). Ainsi, les familles ont besoin d'une aide professionnelle pour prendre certaines décisions concernant la personne ayant une DP (Bond et al., 2003). Ce besoin varie dans le temps, mais est réel et important, et ce, même plusieurs années après l'annonce du diagnostic, étant donné les impacts à long terme de ce dernier (KolakowskyHayner et al., 2001 ; Sinnakaruppan et al., 2001). Une aide professionnelle serait donc toujours nécessaire et bénéfique au-delà de la phase de réadaptation, même dix ans plus tard (Lefebvre et al., 2004; Ergh et al., 2003; Minnes et al., 2000). Ce soutien est primordial, car il est en relation directe avec le fonctionnement familial et représente un moyen puissant de réduire la détresse psychologique du tandem soignant-soigné.

Â long terme, le système familial s'adapte à la DP, mais des impacts concrets persistent dans le temps. Ainsi, bien que les proches aient modifié leur emploi du temps et réorganisé leurs responsabilités familiales et sociales, certaines inquiétudes subsistent, la charge émotionnelle reste importante, les contacts sociaux demeurent moins fréquents et les difficultés financières se manifestent encore régulièrement.

Bien entendu, les besoins des proches d'une personne qui décède rapidement de sa DP seront différents à long terme par rapport à ceux des proches d'une personne qui survit longuement avec des incapacités physiques. Ainsi, bien que l'issue de certaines DP soit la mort, tant que la personne vit avec sa DP, les besoins de ses proches correspondent à ceux décrits plus avant. Ils deviennent différents uniquement une fois que la mort est imminente ou survenue. Il faut noter à ce sujet que plusieurs diagnostics annonçant une maladie dégénérative n'entraînent pas une mort immédiate. Par exemple, les gens atteints de sclérose en plaques peuvent vivre plusieurs années avec leur maladie - trente à cinquante ans.

\section{LE PROCESSUS DE DEUIL}

Le deuxième processus décrit dans le présent article concerne le deuil. Bien que ce dernier soit habituellement associé à la mort de quelqu'un, un processus apparenté est observé dans d'autres situations de perte, tel un diagnostic de DP. En effet, en plus de faire traverser les étapes que nous venons de décrire, l'annonce d'un diagnostic de DP demande aux membres du système familial de faire le deuil d'une partie de leurs rêves et de leurs attentes face à la personne atteinte. Pour cette raison, à l'annonce du diagnostic de DP, parfois même avant, le processus de deuil s'entame. Ce processus se définit comme étant un mécanisme psychologique qui permet à la personne vivant la perte de se réorganiser intérieurement pour s'adapter à l'événement et ensuite réorienter sa vie. Ce processus permet à la personne de se détacher affectivement de l'objet investi pour être capable ultérieurement de réinvestir de nouveaux attachements (Lefebvre et al., 2005).

Kübler-Ross (1981) décrit le deuil comme étant un processus comportant six étapes : le choc initial, le déni, la révolte, le marchandage, la tristesse et l'acceptation. Par contre, elle spécifie que les personnes endeuillées ne vivent pas toutes les étapes dans le même ordre et que certaines personnes ne vivent pas les six étapes, bien qu'elles en vivent toujours au moins deux. Pelchat et ses collègues (2005) ainsi que Lefebvre et ses collègues (2005) ont bonifié ce modèle en en proposant un en huit phases interreliées à travers lesquelles la personne endeuillée chemine : l'appréhension, le choc de l'annonce, la dénégation et le déni, la colère, la culpabilité, la dépression, l'adaptation et la transformation. La spécificité de ce modèle est son caractère dynamique et systémique se situant dans une conception évolutive (figure 1 : Le processus de deuil). Il est dynamique puisque plusieurs phases peuvent coexister et des va-et-vient sont possibles entre les différentes phases. Il est systémique étant donné que les membres du système familial s'interinfluencent dans leur cheminement. Aussi, la conception se veut évolutive, car le commencement d'une étape du processus sous-entend la résolution de la phase précédente. Nous présenterons brièvement ici les particularités de chacune des phases de ce modèle.

\section{PHASE 1 : L'APPRÉHENSION}

L'appréhension se produit avant même que le diagnostic de DP soit connu. Elle représente en fait le moment où la famille reconnaît que la personne a des difficultés, mais ne sait pas de quelle origine ni à quoi 


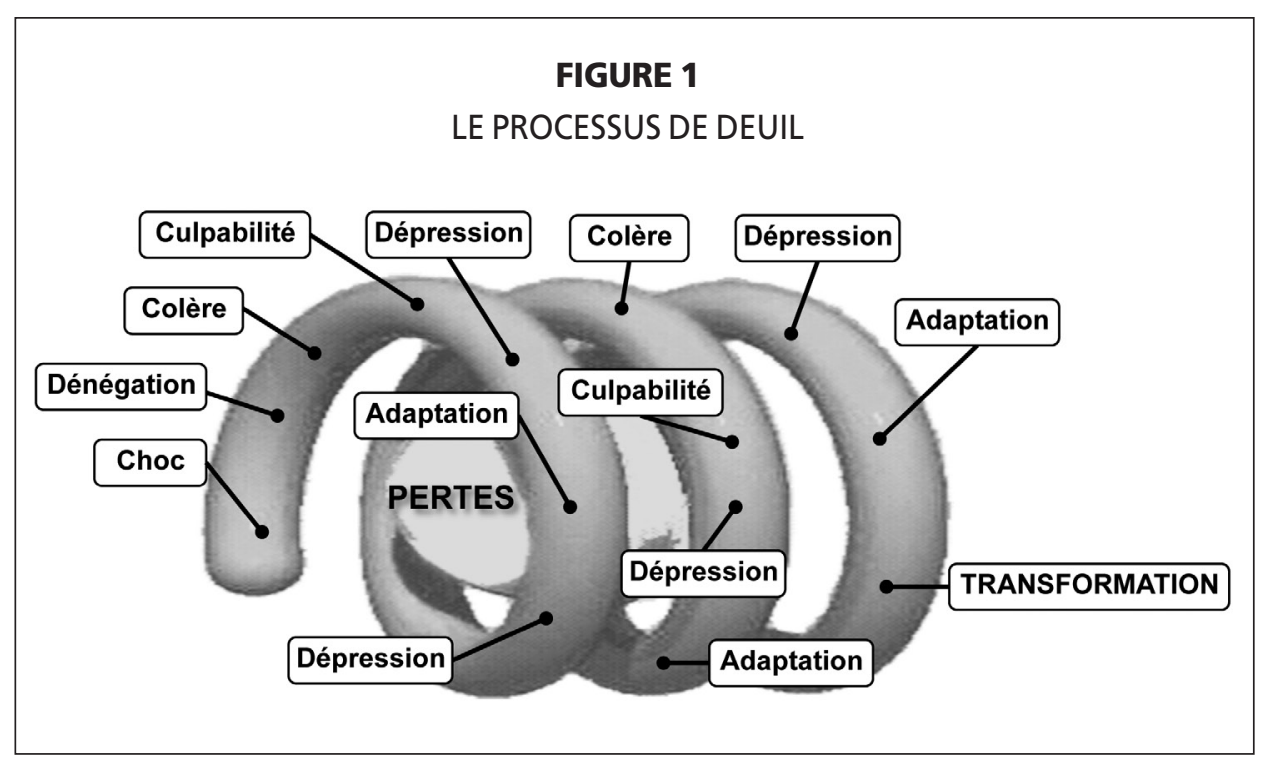

Source: Hélène Lefebvre, Ph. D. et Marie Josée Levert, Ph.D. (cand.),

Équipe de recherche interdisciplinaire sur la famille (ÉRIFAM), Université de Montréal.

s'attendre. Cette phase a comme fonction de préparer la famille au choc de l'annonce de l'événement.

\section{PHASE 2: LE CHOC DE L'ANNONCE}

L'annonce arrive comme un choc et paralyse le fonctionnement du système familial et le fonctionnement psychique de chacun de ses membres. Il correspond à la deuxième étape du processus d'adaptation décrit plus avant, nous ne le détaillerons donc pas plus ici.

\section{PHASE 3: LA DÉNÉGATION ET LE DÉNI}

Une fois le choc initial passé, une montée d'angoisse est vécue. Pour s'en protéger, le psychisme des proches endeuillés met en place un mécanisme de défense qui est la dénégation, soit le fait de se dire que l'événement n'a pas pu se produire. Certaines recherches (Cohadon, 2000; Doka, 1989) démontrent à ce sujet que, étant donné que la personne ayant une DP est encore présente physiquement à l'inverse d'un deuil faisant suite à un décès, ses proches resteront plus longtemps en période de dénégation, ayant plus de difficulté à identifier l'objet réel de la perte. Si ce mécanisme s'avère insuffisant pour contenir l'angoisse, un déni de la réalité peut alors se mettre en place. Ce mécanisme moins adaptatif conduit à un rejet de la réalité et à la construction d'une néoréalité où la DP n'existe pas.

\section{PHASE 4: LA COLÈRE}

La diminution progressive du déni conduit à une reprise graduelle du contact avec la réalité et s'exprime par des réactions de révolte et de colère dirigée vers l'extérieur, que ce soit contre la personne atteinte ou tout autre acteur ou facteur pouvant être tenu responsable de la DP.

\section{PHASE 5 : LA CULPABILITÉ}

Cette colère, surtout celle contre la personne atteinte, peut être impossible à exprimer pour les proches. Elle sera donc dirigée vers d'autres cibles, souvent le proche lui-même, qui développera de la culpabilité face à l'état de la personne atteinte et la considérera comme un reproche vivant. À cette phase, de la surprotection s'observe souvent.

\section{PHASE 6 : LA DÉPRESSION}

Après avoir pris contact avec ses émotions de colère et de culpabilité, le proche commence graduellement à exprimer sa tristesse, voire sa dépression, et à se centrer sur son monde intérieur. Cette période constructive marque le début de l'intégration de la nouvelle situation en amenant le proche à réfléchir sur sa vie.

\section{PHASE 7: L'ADAPTATION}

Lorsqu'il arrive à la phase de l'adaptation, le proche apprend à vivre avec les incapacités causées par la DP et leurs impacts sur sa vie quotidienne. Il apprend à reconnaître les compétences de la personne. C'est à ce moment que le système familial réinvestit la personne telle qu'elle est avec sa DP.

\section{PHASE 8: LA TRANSFORMATION}

Plusieurs modèles proposés pour refléter le processus de deuil se terminent avec l'adaptation du système familial à la perte vécue. Par contre, le présent modèle rappelle que le travail de deuil insère les personnes et les familles dans un processus d'apprentissage qui les transforme. Le processus se termine donc par la phase de transformation, qui représente la capacité de la famille à se sentir compétente et confiante pour utiliser ses ressources et ses savoir-faire. Ce changement se fait sentir de l'intérieur tant sur le plan des valeurs, des croyances que des connaissances.

\section{PARTICULARITÉS DU PROCESSUS DE DEUIL SUIVANT L'ANNONCE D'UNE DÉFICIENCE PHYSIQUE}

Ces huit phases sont influencées par différents facteurs particuliers à la DP. Ainsi, il faut d'abord savoir que le processus de deuil qui accompagne une DP peut n'être jamais achevé. En effet, le caractère évolutif de la DP - vers le mieux-être ou l'inverse - sous-entend des annonces répétées de pertes, ou encore la montée de l'espoir, se concluant souvent par un nouveau désespoir. Cela fait en sorte que le processus est récurrent. Par exemple, lorsque la DP entraîne la mort, plusieurs chocs ont lieu; l'annonce du diagnostic, suivie par l'annonce répétée de pertes significatives - perte de la capacité de marcher, perte de la capacité d'utiliser la toilette, etc. jusqu'à ce que la mort survienne comme ultime choc et déstabilise une nouvelle fois le système familial. Le processus de deuil reprend à chaque choc, à chaque perte. Par contre, le processus peut tant être ralenti par les difficultés non résolues qu'avaient causées les chocs précédents, qu'être facilité par les ressources personnelles, familiales et sociales développées à la suite de la confrontation aux chocs précédents.

En plus d'être récurrent, le deuil qui fait suite à l'annonce d'un diagnostic de DP présente des particularités que peuvent éclairer les concepts de deuil ambigu (Boss, 1999), de deuil non affranchi (St-Charles, 1998; Doka, 1989) et de deuil psychosocial (Doka, 1989). Le deuil ambigu suit un événement où les faits ne sont pas clairs ou non disponibles et où chacun donne une signification différente à l'événement. Par exemple, après un accident de la route, lorsqu'il est impossible de savoir si l'accidenté pourra marcher à nouveau ou non, l'ambiguïté fait en sorte que les proches ne savent pas s'ils doivent faire le deuil de la marche ou non.

Pour sa part, le deuil non affranchi représente l'expérience des personnes endeuillées dont les droits, les rôles et les capacités d'être en deuil ne sont pas reconnus socialement. Par exemple, les proches de personnes ayant une DP se font parfois dire qu'ils ne devraient pas être tristes puisque la personne atteinte est encore en vie. Étant donné que la perte est partielle (des pertes existent, mais la personne est encore là), la douleur et le deuil qui y sont rattachés ne sont pas reconnus socialement.

En ce qui concerne le deuil psychosocial (Doka, 1989), il s'agit de ce qui suit une perte psychocognitive. Par exemple, 
il peut s'agir de changements - fréquents chez les personnes ayant une DP avec atteintes neurologiques - au niveau de la personnalité, ou des facultés cognitives et comportementales qui font en sorte que le système familial devra reconstruire sa représentation psychosociale du proche atteint.

\section{LES ISSUES DE CES PROCESSUS}

En regardant le processus de deuil tel que décrit par Pelchat et ses collègues (2005) ainsi que Lefebvre et ses collègues (2005), il a été possible de voir que son issue n'est pas la simple acceptation, malgré ce que proposait KüblerRoss (1981). Le système familial s'adapte et se transforme à cette nouvelle réalité. Il transforme ses habitudes, son modèle relationnel, pour faire face aux obstacles devant lui. La résilience s'observe entre autres par cette transformation.

Mais, qu'est-ce que la résilience? Plusieurs auteurs se sont penchés sur sa définition. Certains la définissent comme une série de caractéristiques personnelles, d'autres comme l'issue positive d'une crise, d'autres comme un processus, et d'autres encore comme l'ensemble de ces trois composantes. Dans le présent texte, le modèle de Richardson et ses collègues (1990) sera utilisé. Selon eux, le processus résilient, facilité par certaines caractéristiques personnelles, permet une réintégration résiliente. Leur modèle propose quatre issues possibles à un événement difficile, comme l'est l'annonce d'un diagnostic de DP, soit une réintégration dysfonctionnelle, mésadaptée, homéostatique ou résiliente (Figure 2: Issues possibles des processus d'adaptation et de deuil).

La réintégration dysfonctionnelle renvoie à un mode d'adaptation qui ne permet pas à l'individu ou au système familial de fonctionner correctement. La réintégration mésadaptée renvoie à l'idée que le système fonctionne moins bien qu'avant l'événement difficile. La réintégration homéostatique fait référence à un retour au niveau de fonctionnement équivalent, ce qui ne veut pas dire identique, à celui d'avant. La réintégration résiliente définit les systèmes qui arrivent à développer un niveau de fonctionnement supérieur à celui qu'ils avaient auparavant, grâce ou à la suite de l'événement traumatique.

L'utilisation de comportements résilients permet donc aux membres du système familial et au système lui-même de s'adapter à cette situation difficile. Dans une famille, la résilience se joue tant au niveau individuel que familial. La résilience individuelle consiste en la capacité de chacun à se prendre en charge, à être proactif et à s'engager dans le processus

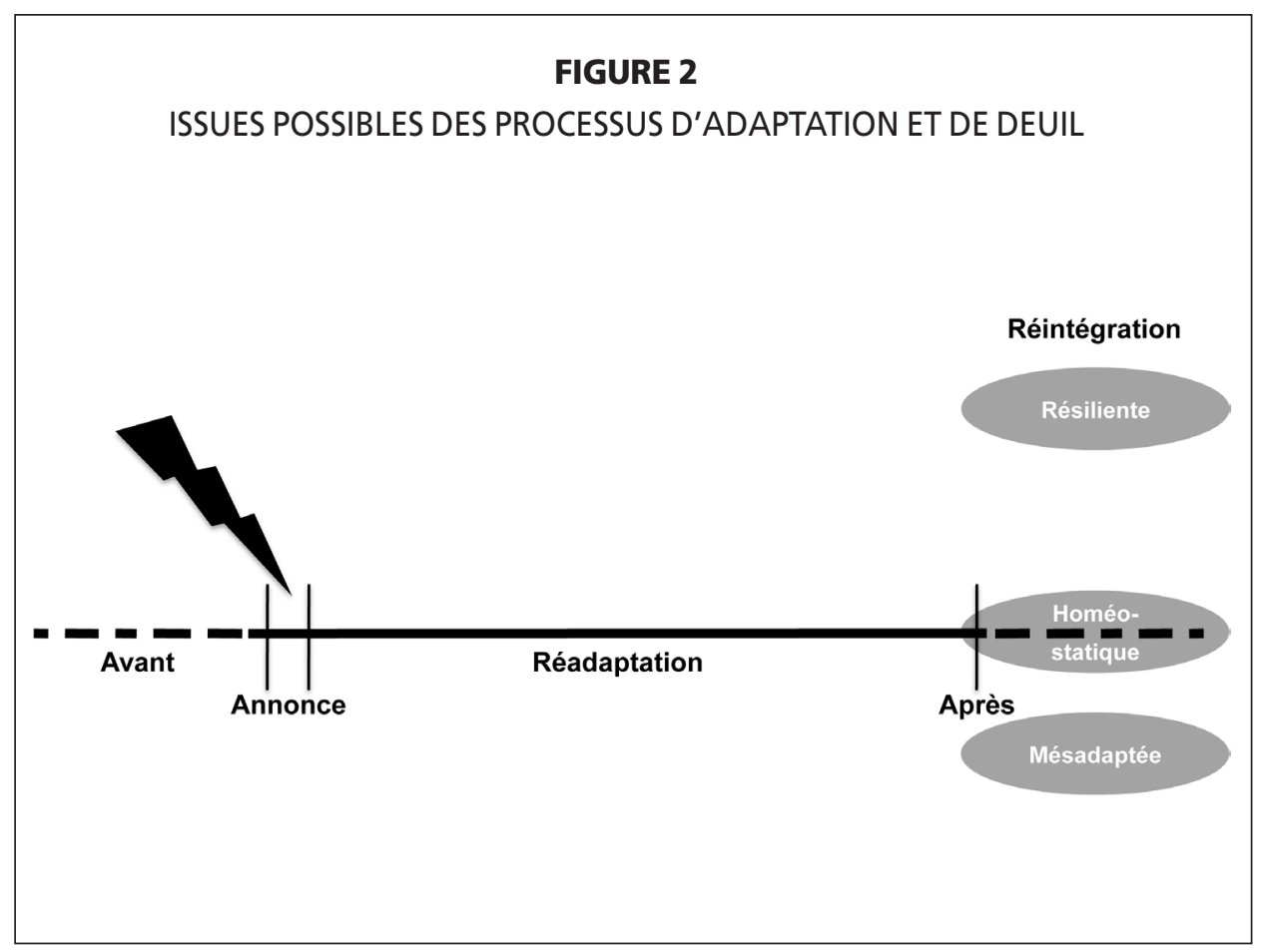

de transformation individuelle et familiale pour faire face à la situation de stress, lui donner un sens constructif et permettre à chacun de trouver un nouvel équilibre pour lui-même afin de participer au renouvellement du bien-être familial (Pelchat et al., 2005). Pour sa part, la résilience familiale consiste en «la capacité développée au sein d'une famille, elle-même éprouvée, à soutenir et aider un ou plusieurs de ses membres victimes de circonstances difficiles ou à construire une vie riche et épanouissante pour chacun de ses membres, malgré un environnement adverse auquel l'ensemble est soumis » (Delage, 2008, p. 78-79).

Ces deux types de résilience permettent un retour à l'équilibre et l'apprentissage de la capacité à rebondir, à se reconstruire et à reprendre le contrôle sur sa vie. Quand cela n'est pas fait dès le départ, elle permet aussi d'accorder un sens positif à l'événement traumatisant en lui trouvant une raison d'être (mission) ou en y découvrant des gains et des bénéfices a posteriori (apprentissages).

\section{LA RÉSILIENCE}

Certains auteurs (Waller, 2001; Richardson, 1990) ont identifié des facteurs de protection pouvant jouer un rôle dans la capacité d'être résilient pour certaines personnes ou systèmes. Ces facteurs peuvent être individuels, comme être positif et confiant en soi, familiaux, comme la bonne entente des parents et la responsabilisation des enfants pour certaines tâches de la maison, ou communautaires, comme la sécurité du quartier et la présence de services adéquats. Bien que la présence ou l'absence de ces facteurs ne permettent pas de prédire avec exactitude quelles personnes et quels systèmes pourront être résilients, il semble qu'ils permettent de favoriser la résilience. En contrepoids de ces facteurs de protection, il y a ce que certains (Waller, 2001; Richardson, 1990) nomment les facteurs de risque. Tout comme les facteurs de protection, les facteurs de risque peuvent être individuels, familiaux ou communautaires.

Les écrits indiquent que ces différents facteurs se conjuguent de deux façons pour favoriser ou entraver le processus de résilience. Delage (2008) parle de la balance de vulnérabilité. Selon cette théorie, le fait d'avoir plus de facteurs de protection favoriserait le processus de résilience, alors que l'inverse l'entraverait. Toutefois, une deuxième relation entre ces facteurs entre aussi en jeu. En effet, Waller (2001) propose l'image de l'effet de vagues (ripple effect) pour expliquer qu'un facteur isolé peut prendre une ampleur plus ou moins importante, selon le contexte. Comme une pierre jetée dans l'eau, les vagues qu'un élément crée peuvent prendre de l'ampleur si rien ne les interceptent. Ainsi, peu importe le nombre réel de facteurs de risque par rapport à ceux de protection, leur importance relative pour l'individu et le système familial est ce qui aurait le plus d'impact sur la capacité de rebondir.

\section{CONCLUSIONS POUR L'INTERVENTION}

Nous savons donc maintenant que les systèmes familiaux traversent un processus linéaire d'adaptation en même temps 


\section{FIGURE 3}

PROCESSUS DE DEUIL DANS LA CHRONOLOGIE DU PROCESSUS D'ADAPTATION

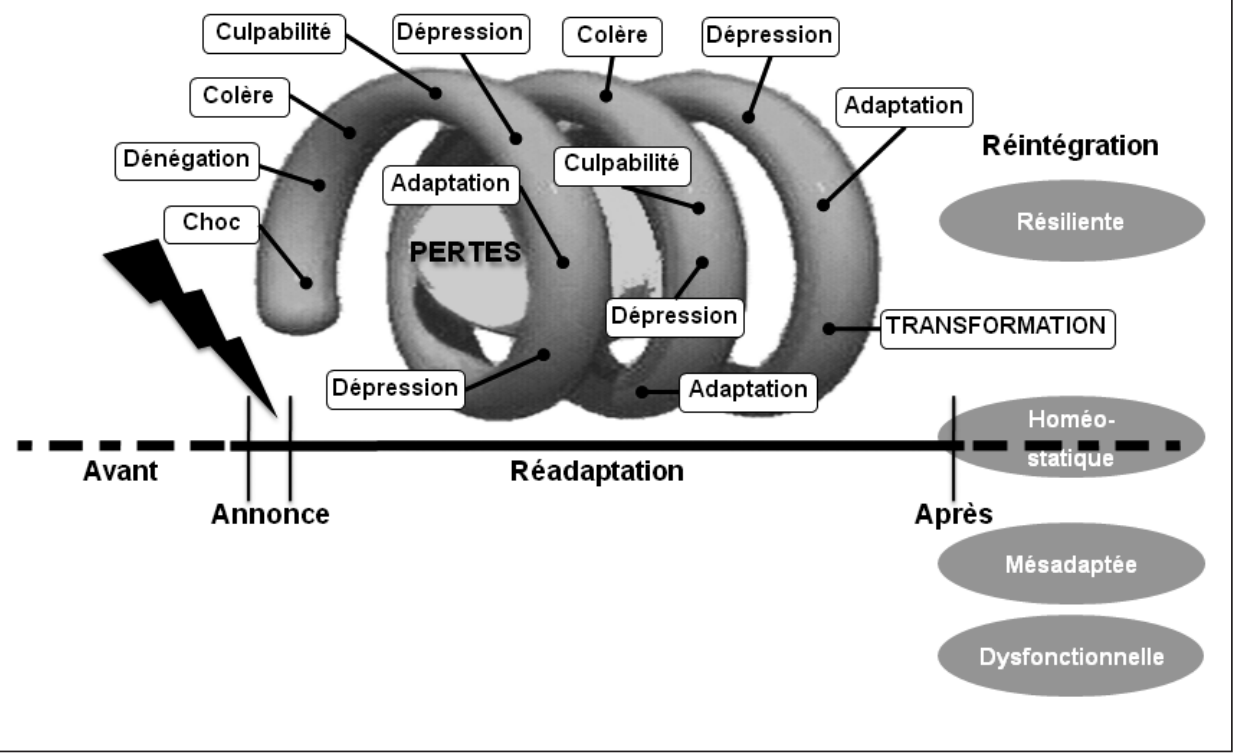

qu'ils en traversent un non-linéaire de deuil. Comme nous l'avons vu, ces processus peuvent avoir différentes issues, selon les facteurs de protection et les facteurs de risques qui entrent en jeu (Figure 3 : Processus de deuil dans la chronologie du processus d'adaptation).

Ces données nous permettent de nous demander ce que nous pouvons faire comme intervenant pour favoriser la résilience des familles. Une partie de la réponse vient du concept de «tuteur de résilience» (Delage, 2008; Cyrulnik, 1999). Un tuteur de résilience est une personne «capable d'accueillir la souffrance et d'aider à reprendre le cours de la vie» (Delage, 2008, p. 22). En devenant un tuteur de résilience, on permet aux personnes en difficulté de grandir à travers l'épreuve qu'elles vivent. Des petits gestes pouvant sembler anodins, comme prendre le temps d'expliquer à un enfant comment se forment les nuages, peuvent prendre une signification très importante pour la personne. C'est la multiplication de ces gestes, ces petites attentions bien placées et non calculées, qui peuvent servir de tremplin à la résilience. Malheureusement, bien que plusieurs pistes aient été identifiées pour déterminer comment devenir tuteur de résilience, aucune n'a de résultats assurés. Une personne peut être un tuteur de résilience sans même le vouloir. Ainsi, la volonté de jouer ce rôle n'est pas synonyme de pouvoir le faire.

Malgré la difficulté à identifier une façon d'amener un système familial à être résilient, plusieurs pistes d'intervention peuvent servir à bien accompagner une famille à travers une crise. Par exemple, on peut impliquer les proches dans la démarche, reconnaître leur expertise, les informer, leur offrir du soutien, cerner leurs attentes et tenter autant que possible d'y répondre, les encourager à bâtir un réseau de support, explorer leurs projets de vie à moyen et à long terme, respecter les valeurs et les croyances du système familial, mettre l'accent sur leurs forces, respecter leurs limites et les nôtres, référer au besoin, viser de petits changements, préparer la fin des interventions et les informer des ressources disponibles à court, moyen et long terme (réseautage) (Lefebvre et al., 2007).

En résumé, les interventions pouvant favoriser la résilience des familles restent à définir et à explorer. En attendant que cela soit fait, un travail de collaboration peut être réalisé avec les membres du système familial pour développer leurs facteurs de protection et minimiser leurs facteurs de risques. Devenir tuteur de résilience n'est pas chose simple, ni même planifiable. Par contre, devenir partenaire dans un moment difficile est réalisable et, qui sait, peut-être deviendrez-vous ainsi la personne qui permet aux membres du système familial déstabilisé de grandir dans l'adversité.

\section{Bibliographie}

BOND, E., C. DRAEGER, B. MANDELCO et M. DONNELLY (2003). «Needs of family members of patients with severe traumatic brain injury. Implications for evidencebased practice», Critical Care Nursing, vol. $23, n^{\circ} 4$, p. 63-72.
BOSS, P. (1999). Ambiguous Loss, Cambridge, MA, Harvard University Press.

COHADON, F. (2000). Sortir du coma, Paris, Odile Jacob.

COULTER A. (1998). «Evidence based patient information", British Medical Journal, vol. 317, p. 225-226

CYRULNIK, B. (1999). Un merveilleux malheur, Paris, Odile Jacob.

DEGENEFFE, C.E. (2001). «Family caregiving and traumatic brain injury ", Health and Social Work, vol. 26, n ${ }^{\circ}$ 4, p. 257-268.

DELAGE, M. (2008). La résilience familiale, Paris, Odile Jacob.

DESTAILLATS, J.-M., J.-M. MAZAUX et C. BELIO (2007). Le lien familial à l'épreuve du handicap et des soins, Service de médecine physique et de réadaptation du Dr Mazaux, CHU Pellegrin, France, en ligne, <http:// www.gfrup.com/gfrup_handicap_lien_ famille.htm>, consulté le 2010-07-26.

DOKA, J.C.L. (1989). Disenfranchised Grief: Recognizing Hidden Sorrow, Toronto, Lexington Books.

ERGH, T.C., R.A. HANKS, L.J. RAPPORT et R.D. COLEMAN (2003). "Social support moderates caregiver satisfaction with life following TBI », The Journal of Clinical and Experimental Neuropsychology, vol. 25, $\mathrm{n}^{\circ} 8$, p. 1090-1101.

FALLOWFIELD, L.J., V.A. JENKINS, H.A. BEVERIDGE (2002). "Truth may hurt but deceit hurts more: Communication in palliative care», Palliative Medicine, vol. 16, p. 297-303.

KOLAKOWSKY-HAYNER, S.A., K.D MINER et J. KREUTZER (2001). "Longterm life quality and family needs after traumatic brain injury ", Journal of Head Trauma Rehabilitation, vol. 16, n ${ }^{\circ}$, p. 374-384.

KÜBLER-ROSS, E. (1981). Living with Death and Dying, New York, Macmillan.

LACERTE, J. et K. TAUPIER (2002). TANDEM - Un cahier de bord destiné aux proches des personnes ayant subi un traumatisme crânio-cérébral (TCC) ou un accident vasculaire cérébral (AVC), Montréal, Centre de réadaptation Lucie-Bruneau.

LEFEBVRE, H., G. CLOUTIER et M.J. LEVERT (2007). "Dix ans après avoir subi un traumatisme crânien: participation sociale et continuum de services ", Intervention, vol. 127, p. 99-109.

LEFEBVRE, H., C. DAVID, I. GÉLINAS, D. PELCHAT, B. SWAINE, C. DUMONT, B. MICHALLET, P. BOUDREAULT (2007). L'adéquation entre les besoins vécus par les proches de personnes ayant un traumatisme craniocérébral et les services offerts par le continuum de soins, en collaboration avec M.J. LEVERT, G. CLOUTIER et J. GAUVIN-LEPAGE, Montréal, Université de Montréal (MSSS, REPAR, SAAQ, 20052007), $70 \mathrm{p}$.

LEFEBVRE, H. et M.J. LEVERT (2005) "Traumatisme craniocérébral: de la souffrance à la résilience», Frontières, vol. 17, $\mathrm{n}^{\circ} 2$, p. 53-58. 
LEFEBVRE, H., M. VANIER, B. SWAINE, É. DUTIL, M. PÉPIN, P. FOUGEYROLLAS, et al. (2004). La participation sociale à long terme des personnes ayant subi un traumatisme crânien et l'impact chez les proches, 10 ans post-traumatisme, Montréal, Université de Montréal.

MANN, D.W. (2002). "Family caregivers' reactions and coping for persons with brain injury ", Brain injury, vol. 16, $\mathrm{n}^{\circ} 12$, p. 1025-1037.

MINNES, P., S. GRAFFI, M.L. NOLTE, P. CARLSON et L. HARRICK (2000). «Coping and stress in Canadian family caregivers of persons with traumatic brain injuries", Brain Injury, vol. 14, $\mathrm{n}^{\circ}$ 8, p. 737-748.

O'CONNELL, B. et L. BAKER (2004). «Managing as carers of stroke survivors: Strategies from the field", International Journal of Nursing Practice, vol. 10, $\mathrm{n}^{\circ} 3$, p. $121-126$.
PELCHAT, D. et H. LEFEBVRE (2005). Apprendre ensemble, Le PRIFAM, Programme d'intervention interdisciplinaire familial, Montréal, Chenelière - McGrawHill.

RICHARDSON, G.E., B.L. NEIGER, S. JENSEN et K.L. KUMPFER (1990). «The resiliency model», Health Education, vol. $21, \mathrm{n}^{\circ} 6$, p. 33-39.

ROCHETTE, A., N. KORNER-BITENSKY et J. DESROSIERS (2007). "Actual $v$ s best practice for families post-stroke according to three rehabiliation disciplines ", Journal of Rehabilitation Medicine, vol. 39, p. 513519.

ROLLAND, J.S. (1994). Families, Illness and Disability: An Integrative Treatment Model, New York, Basic Books.

SINNAKARUPPAN, I. et D.M. WILLIAMS (2001). "Family carers and the adult headinjured: A critical review of carers' needs ", Brain Injury, vol. 15, no 8, p. 653-672.
ST-CHARLES, D. (1998). "La reconstitution identitaire des "proches-soignants" des personnes traumatisées cranio-cérébrales", dans J.C. KALUBI, B. MICHALLET, N. KORNER-BITENSKY et S. TÉTREAULT (dir.), Innovations, apprentissages et réadaptation en déficience physique, Montréal, IQ Éditeur, p. 97-103.

WALLER, M.A. (2001). "Resilience in ecosystemic context: Evolution of the concept », American Journal of Orthopsychiatry, vol. 71, n 3, p. 290-297.

WILES, R. et al. (1998). «Providing appropriate information to patients and carers following a stroke», Journal of Advanced Nursing, vol. 28, n 4, p. 794-801.

\section{Note}

1. L'auteure du présent texte tient à souligner l'importance de l'apport des travaux de la professeure Hélène Lefebvre, qui ont particulièrement été pertinents pour le sujet traité ici. 